\title{
Editor's Note: Introducing BAPcast (Behavior Analysis in Practice: The Podcast)
}

\author{
Stephanie M. Peterson ${ }^{1}$
}

Published online: 26 April 2021

(C) Association for Behavior Analysis International 2021

As the incoming editor in chief of Behavior Analysis in Practice (BAP), one of the most immediate initiatives I wanted to get started on was the creation of BAPcast, a podcast based on the articles published in the most recent issues of the journal. There were several reasons I was eager to get this project off the ground. First, I felt it was important for the journal to have some presence on multimedia platforms. People seem to enjoy being able to access information in a variety of different ways and not just through print. I believe it is important for $B A P$ to make information available on such alternative platforms to stay relevant and easily accessible. Second, many practitioners who might read $B A P$ likely spend a lot of time in the car, driving to see clients. Making information available in an audio format might allow these individuals to multitask and use drive time efficiently to stay abreast of current research and behavior-analytic content. Third, folks who are publishing in $B A P$ are really amazing professionals and have an incredible amount of expertise to offer the field. These individuals are at the forefront of identifying creative solutions to applied, practical problems in the world using principles of behavior analysis as their foundation. Although the articles published in the journal do a great job of highlighting these potential solutions by conceptualizing problems in a behavior-analytic framework, describing the procedures in place during the studies, describing how the behaviors targeted for assessment and intervention were defined and measured, displaying the results in meaningful ways, and analyzing those results in a behavior-analytic framework, what the articles often do not highlight are all the "behind-the-scenes" steps the authors had to take in order to execute the study or to develop their ideas. Very often, these backstories are very interesting, and learning more about the

Stephanie M. Peterson

stephanie.peterson@wmich.edu

1 Department of Psychology, College of Arts and Sciences, Western Michigan University, Mail Stop 5439, 3740 Wood Hall, Kalamazoo, MI 49008-5439, USA backstories can help readers have a deeper understanding of the issues and obstacles the authors faced, the process they took to overcome these obstacles, the related ideas they brought to bear on the problem, the literature they studied, and the people with whom they collaborated to see the project through. It is my hope that giving the authors a platform to talk about these backstories will be informative for the readership of $B A P$ and might help practitioners further develop their analytical skills or publish in BAP themselves (or both!).

I would like to introduce you to Dr. Cody Morris, who is an assistant professor at Salve Regina University and the host of the podcast. Each time a new issue of BAP is published, Dr. Morris and I work together to identify the articles published in that issue that we think will lend themselves well to a podcast episode. We make our selections based on the level of interest we think the readers will have in the topic, the quality of the work described in the article, and the like. Then, Dr. Morris works with his graduate students to do a great deal of research and preparation for an informative podcast. Dr. Morris then contacts the authors to see if they are willing and able to have a recorded discussion with him. If the authors agree, Dr. Morris records and produces the podcast. We wish we could have podcasts for every article in every issue of $B A P$; however, there are not enough hours in the day for us to produce that many podcasts. We hope we are successful in selecting the articles readers would most like to hear about. You, the readers, can help us with this task. If you have not done so already, please follow the BAP Facebook page (https://www. facebook.com/BApractice). When new episodes of the podcast are released, we will make an announcement on this page. We would also like to engage the listening audience regarding their ideas and questions for $B A P$ authors. We may be posting some queries on the Facebook page. If you are following the page, we would love it if you would comment regarding articles or authors you would like to know more about, as well as questions you would like to ask the authors. This will help us ensure the podcast is providing content that is most interesting and helpful to you. 
You can subscribe to the podcast on your favorite podcast application by searching "Behavior Analysis in Practice: The Podcast." (Also, Spotify and iTunes links are found at the end of this editor's note.) At the time of this publication, the first season has already been partially released, with multiple episodes available for your listening pleasure. More are on the way. We anticipate having approximately 10 episodes per season and at least two seasons per year. We hope you view the creation of BAPcast as an interesting, engaging, and informative complement to the print version of the journal. We hope the podcast enhances your professional development in behavior analysis. We recognize that it is difficult to keep up with professional reading and hope that BAPcast allows you to access your professional reading in another medium and creates a motivating operation for prioritizing time for professional reading, especially reading $B A P$. We welcome your feedback, both positive feedback about what you like and constructive criticism regarding how we can improve the podcast over time. I hope you will join me in welcoming Dr. Morris to the editorial team at BAP and thank him and his graduate students for their extra efforts in helping create and maintain BAPcast.

Finally, I would be remiss if I did not recognize Dr. Jim Carr for his contributions to the podcast. Dr. Carr is an incredibly talented musician. He and his musical partners, known as the band New Latitude, graciously allowed us to use one of their tracks as the background music for the podcast. If you want to hear more of New Latitude's music, please visit http:// www.newlatitudemusic.com.

In closing, I look forward to my term as editor in chief for $B A P$ over the next few years. I would like to extend a very heartfelt thank-you to Dr. Jonathan Tarbox, the outgoing editor in chief. Dr. Tarbox served the journal well for the past 3 years. I appreciate his good stewardship during that time. I also appreciate all the extra efforts he has made to make the transition from his term to mine as smooth as possible. There are several special issues coming to fruition soon, thanks to Dr. Tarbox's efforts during his term. He and I will be working together to see those through. Please visit the $B A P$ website (see the following links) to learn more about those. I hope to have a call for papers or two coming soon for some additional special issues. In addition, I will be working with the editorial board of the journal to discuss other initiatives. In the meantime, I hope you find the current issue informative and that it furthers your practice of behavior analysis.

Subscription links for Behavior Analysis in Practice: The Podcast

Spotify: https://open.spotify.com/show/ 4 P c 2 dhtk CX 7 aWbUsA O 1 htR?si=d2 Ct $1 \mathrm{na}-$ S7WUWHjO1k0sqA

Apple Podcasts: https://podcasts.apple.com/us/podcast/ behavior-analysis-in-practice-the-podcast/id1555960276

Behavior Analysis in Practice Website:

https://www.springer.com/journal/40617?gclid= Cj0KCQjw3duCBhCAARIsAJeFyPXA0gyVMIz02oABJP K G 8 T 1 N D n W y U C r Z K tv W Z fC V C s u 1 U oownu9QaAhcAEALw wcB

Publisher's Note Springer Nature remains neutral with regard to jurisdictional claims in published maps and institutional affiliations. 